\title{
ARBITRARIEDAD Y MOTIVACIÓN EN LAS COLOCACIONES
}

\author{
ARBITRARINESS AND MOTIVATION IN COLLOCATIONS
}

\author{
INMACULADA PENADÉS MARTÍNEZ \\ Universidad de Alcalá \\ inmaculada.penades@uah.es
}

\section{RESUMEN}

En este artículo se examina la concepción de la arbitrariedad y la motivación en el ámbito de las colocaciones. Los autores que caracterizan estas unidades con la propiedad de la arbitrariedad no ofrecen una definición del término y, en general, la vinculan con el uso lingüístico, que determina las restricciones de selección del colocativo por parte de la base. El concepto de motivación tampoco es definido al aplicarse a las colocaciones, aunque en la actualidad se prefiere esta propiedad para su caracterización y no la de arbitrariedad. Desde esta perspectiva, las colocaciones se consideran motivadas porque un colocativo no es una elección individual de una base; al contrario, las bases forman clases semánticas y presentan algún rasgo, generalmente semántico, compartido con el colocativo. Sin negar este hecho, en el artículo se parte de la concepción de Saussure sobre la arbitrariedad y de una definición de motivación entroncada con el estructuralismo europeo. Se concluye que todas las locuciones son relativamente arbitrarias, es decir, parcialmente motivadas y que presentan los tipos de motivación morfológica y semántica. Esta visión teórica de las colocaciones tiene implicaciones aplicadas en el ámbito de la lexicografía y de la enseñanzaaprendizaje de la lengua.

Palabras clave: Colocaciones, locuciones, arbitrariedad, motivación, F. de Saussure, estructuralismo, historiografía lingüística.

\section{ABSTRACT}

In this article we examine the concept of arbitrariness and motivation in the area of collocations. The authors who characterise these units as having the property of arbitrariness do not offer a definition of the term, and generally link it to linguistic usage, which determines the limits in the choice of a collocative by the base. Neither is the concept of motivation defined on applying it to collocations, although this concept is currently preferred for their characterisation, in preference to arbitrariness. From this 
standpoint, collocations are considered to be motivated because a collocative is not an individual choice made by a base; on the contrary, bases form semantic classes and show certain features, usually semantic ones, which are shared with the collocative. Without wishing to deny this fact, this article starts from Saussure's concept of arbitrariness and a definition of motivation linked to European structuralism. The conclusion is that all idioms are relatively arbitrary, that is to say, partially motivated, and that they present both morphological and semantic motivation. This theoretical vision of collocations has implications that are applied in the field of lexicography and in language teaching and learning.

Keywords: Collocations, idioms, arbitrariness, motivation, F. de Saussure, structuralism, linguistic historiography.

Recibido: 07.04.2017. Aceptado: 29.09.2017.

\section{INTRODUCCIÓN}

$\mathrm{D}$ urante la década de los noventa y en los primeros años del siglo XXI, en el análisis de las colocaciones del español una de las características asignadas por los estudiosos a estas unidades ha sido la arbitrariedad, junto con el binarismo y su pertenencia al plano de la norma. Con el término arbitrariedad se ha hecho referencia a la particularidad del elemento base de una colocación de escoger el colocativo con el que se combina de manera arbitraria, o bien a que las restricciones de combinación entre la base y el colocativo vienen dadas por el uso lingüístico. Con el paso de los años, la postura de los investigadores respecto a la arbitrariedad de las colocaciones se ha ido modificando y, en la actualidad, son ya varios los que sostienen que las colocaciones son motivadas o, al menos, presentan un mayor o menor grado de motivación. Posiblemente, el autor que ha marcado la inflexión conducente a considerar las colocaciones motivadas, y no arbitrarias, ha sido Bosque.

Ahora bien, el estudio de esta cuestión conlleva una serie de aspectos que no quedan completamente aclarados, lo que dificulta avanzar en la solución de un problema complejo. En efecto, en general no se especifica qué se entiende exactamente por arbitrariedad ni por motivación; junto a ello, no se diferencia la perspectiva de análisis adoptada: diacrónica / sincrónica, propia del investigador / específica del hablante; y no se tiene en cuenta que las afirmaciones realizadas sobre las colocaciones tienen un alcance limitado por los propios ejemplos objeto de análisis. Así pues, puede resultar improcedente establecer generalizaciones sobre la caracterización y clasificación de las colocaciones, con el agravante de que su estatus de unidades fraseológicas / unidades no fraseológicas / unidades de la interfaz léxico-sintaxis no queda claramente establecido. 
En consecuencia, en este trabajo se pasa revista a las afirmaciones hechas por los especialistas sobre la arbitrariedad y la motivación de las colocaciones, con el objetivo de determinar si alguna de estas propiedades debe formar parte de la caracterización de unas unidades cuyo análisis sigue siendo objeto prioritario de investigación ${ }^{1}$, tal vez por el retraso con el que esta se inició para el caso del español.

\subsection{La arbitrariedad en las colocaciones}

En el ámbito de las colocaciones, con el término arbitrariedad no se alude a que el lazo que une el significante al significado de un signo lingüístico es arbitrario, es decir, el término en cuestión no se utiliza para dar cuenta del hecho de que la idea correspondiente a un signo no está ligada por ninguna relación interior con la secuencia de sonidos que le sirve de significante, concepción sustentada por Saussure (1916 [1945, p. 130]). Y ello es así en virtud de que una colocación no está constituida por una sola unidad léxica, sino por una combinación de unidades en la que una de ellas, la base, exige la otra, el colocativo, y puesto que, en principio, no parece existir una razón o una causa para tal coocurrencia, se ha considerado que esta es arbitraria, con lo que las colocaciones se han caracterizado con la misma propiedad.

En relación con la lengua española, son varios los autores que, en principio, han mantenido esta posición. En efecto, procediendo cronológicamente, en un primer momento no se menciona la arbitrariedad de las colocaciones de manera explícita, sino de modo indirecto, haciendo referencia al uso lingüístico, que determina las restricciones de combinación que presentan los elementos que forman estas unidades ${ }^{2}$. No hay que olvidar que la vinculación del uso con la arbitrariedad tiene una larga tradición en el ámbito de la Lingüística, pues en la antigua Grecia los anomalistas ya sostenían que la lengua no obedece a verdaderas reglas, y que en ella todo no es más que uso y arbitrariedad (Mounin, 1979, p. 99). Es ejemplo de esta manera de proceder Corpas Pastor (1996, p. 66) cuando afirma que, en función del uso, la base de una colocación determina la elección del colocativo y, además, selecciona en este una acepción especial: dinero negro ${ }^{3}$. También sirve de

${ }^{1}$ Una buena prueba de ello es la reciente monografía sobre colocaciones editada por Torner y Bernal (2017a).

${ }^{2}$ Constituiría una excepción a lo dicho el trabajo de Alonso Ramos (1994-1995), pues, de manera tangencial, se menciona la arbitrariedad al indicar que las funciones léxicas que describen la coocurrencia léxica restringida de las colocaciones lexicalizan ciertos sentidos cuya expresión es arbitraria (Alonso Ramos, 1994-1995: 24). Además, al situar la arbitrariedad en la relación sentidoexpresión, esta autora toma arbitrariedad de un modo que deriva de la concepción de Saussure (1916 [1945: 130]).

${ }^{3}$ La definición de colocación de Corpas Pastor (1996: 66) es: «denominaremos colocación a las combinaciones así resultantes, es decir, a las unidades fraseológicas formadas por dos unidades 
ilustración la concepción de Zuluaga (1998, p. 24) sobre las colocaciones, entendidas como combinaciones regulares de lexemas compatibles entre sí, estabilizadas mediante el uso repetido y convertidas en unidades sintagmáticas de la norma lingüística social: opinión pública .

Con posterioridad, en un monográfico de Lingüistica Española Actual, básicamente dedicado a las colocaciones, García-Page (2001) y Castillo (2001), autores que se hacen eco de la definición de Corpas Pastor (1996), ya se refieren expresamente a la arbitrariedad de las colocaciones, el primero al tratar los adverbios colocacionales, de los que indica (García-Page, 2001, p. 100-101) que el significado intensificador que presentan se debe al uso sancionado por la comunidad lingüística y constituye un valor estereotipado; además, el adverbio que se coloca con un verbo o un adjetivo, formando una colocación, ha sido elegido caprichosamente (arbitrariamente, concreta en la página siguiente García-Page (2001, p. 102)) por la comunidad, que rechaza otros adverbios posibles: oponerse / negar radicalmente, pero oponerse / *negar frontalmente. Por su parte, la segunda autora ya relaciona de manera directa arbitrariedad y uso al considerar que «la arbitrariedad que impera en las restricciones combinatorias impuestas por el uso tradicional es la verdadera responsable de que determinadas combinaciones léxicas constituyan colocaciones en una lengua y no en otra. Es decir, ese carácter arbitrario que se observa en la coaparición de dos elemementos léxicos es el que propicia también la variedad lingüística en el aspecto colocacional» (Castillo, 2001: 139): contraer / pillar / coger I pescar una enfermedad.

Del mismo año que el monográfico de Lingüistica Española Actual, 2001, es la obra de Koike sobre colocaciones léxicas. En ella no se proporciona una definición explícita del término colocación, sino que se facilita una serie de ideas sobre esta clase, organizadas en 1) características formales y semánticas, 2) dinamismo semántico, 3) cohesión semántica, 4) colocabilidad y 5) diferencias entre la estructura sintáctica y la colocacional. De todas ellas, interesa subrayar aquí la primera: características formales y semánticas, relativa a las restricciones combinatorias que sobre las colocaciones impone el uso tradicional, pues tales restricciones se caracterizan por tener cierta arbitrariedad, responsable esta de que determinadas com-

léxicas en relación sintáctica, que no constituyen, por sí mismas, actos de habla ni enunciados; y que, debido a su fijación en la norma, presenta restricciones de combinación establecidas por el uso, generalmente de base semántica: el colocado autónomo semánticamente (la base) no sólo determina la elección del colocativo, sino que, además, selecciona en éste una acepción especial, frecuentemente de carácter abstracto o figurativo». En Corpas Pastor (1998: 42) la restricción combinatoria de las colocaciones se vincula, asimismo, al uso. Por otra parte, la definición de 1996 es retomada en Corpas Pastor (2001: 48).

${ }^{4}$ En este mismo trabajo, Zuluaga (1998: 22) asigna la paternidad de la caracterización de las colocaciones como combinaciones usuales típicas, cuya estabilidad está determinada históricamente por el uso, a Matthias Perl en un artículo de 1975: «Semantische Selektionsbeschränkungen (semantische Valenz) und sprachlich usuell bedingte Restriktionen», publicado en Linguistische Arbeitsberichte, 11 , pp. 48-51. 
binaciones léxicas constituyan colocaciones en una lengua, pero no en otra. La diferencia entre dos lenguas en la selección de los colocativos es la manifestación del carácter arbitrario de las colocaciones (Koike, 2001, p. 215). Otra estudiosa que alude a la arbitrariedad de las colocaciones, a través de la mención a su uso, es Higueras García (2006a), para quien, siguiendo expresamente a Corpas Pastor (1996), estas unidades léxicas ilustran preferencias de combinación y restricciones impuestas por el uso 5 .

No obstante, es la definición del concepto de 'colocación' propuesta por Alonso Ramos (2002a) ${ }^{6}$ la que manifiesta claramente el carácter arbitrario que han ido subrayando otros investigadores, si bien, por la cercanía temporal entre varias de las visiones mantenidas, es difícil establecer si estos han llegado a tal característica en concepciones deudoras de la de Alonso Ramos o de manera independiente. El caso más patente acerca de la dificultad en establecer cuál es la primera caracterización de las colocaciones en español a partir de la propiedad de la arbitrariedad lo constituye el trabajo de Zuluaga (2002a), incluido en el mismo volumen en el que está publicado el de Alonso Ramos (2002a); en aquel se lee que las colocaciones son «combinaciones transparentes, regulares tanto desde el punto de vista semántico como desde el punto de vista gramatical, pero ${ }^{Z}$ con un grado menor o mayor de fijación de sus componentes léxicos, fijación arbitraria, determinada por el uso repetido» (Zuluaga, 2002a, p. 106) ${ }^{8}$. Además, en una obra, también de 2002, que

${ }^{5}$ La misma caracterización se reproduce en Higueras García (2006b), si bien, en trabajos posteriores (Higueras García, 2011), la arbitrariedad queda relegada a favor de la selección léxica de una clase, las bases (correspondientes a argumentos), por parte de los colocativos, que constituyen predicados de tales argumentos (tajantemente se predica de rechazar, oponerse, cerrar, zanjar, separar, etc.), lo que determina que las colocaciones sean transparentes para esta autora. Con total claridad se expresa actualmente al considerarlas ejemplos no arbitrarios de selección léxica (Higueras, 2017).

${ }^{6}$ Para esta autora las colocaciones son «construcciones semi-fraseológicas formadas por dos UL, $\mathrm{L}_{1} \mathrm{~L}_{2}$, en donde $\mathrm{L}_{2}$ es escogida de un modo (parcialmente) arbitrario para expresar un sentido dado y/o un papel sintáctico en función de $\mathrm{L}_{1}$. Así, en error garrafal el adjetivo garrafal es seleccionado para expresar el sentido 'grande' en función de error, ese mismo sentido será expresado por supina, si se predica de ignorancia, por ejemplo» (Alonso Ramos, 2002a: 67). Idéntica definición, solo que con la aparición de la palabra semi-idiomáticas en vez de semi-fraseológicas, se encuentra en Alonso Ramos (2002b: 49). Obsérvese que el hecho de calificar la arbitrariedad de parcial, en estas definiciones, no supone hablar de motivación. Aunque arbitrariedad y motivación son fenómenos tradicionalmente vinculados en los estudios lingüísticos (Penadés Martínez y Díaz Hormigo, 2008), en este caso el carácter relativo de la arbitrariedad no implica una motivación gradual correlativa.

${ }^{7} \mathrm{El}$ subrayado es de Zuluaga.

${ }^{8}$ Con todo, como la publicación de Alonso Ramos (2002b) corresponde a la comunicación presentada al IV Congreso de Lingüistica General, celebrado en Cádiz en 2000, su definición de colocación puede considerarse anterior a la de Zuluaga (2002a). Por otra parte, del mismo año, 2002, es otro trabajo de Zuluaga (2002b), en el que, pese a llevar idéntico título que el de (2002a) y pese a coincidir en gran parte del contenido, este autor facilita una definición de colación en la que ya no hay referencia explícita a la particularidad de la arbitrariedad: «Las llamadas colocaciones se definen como combinaciones de por menos (sic.) dos lexemas en relación hipotáctica, fijadas (estabilizadas, consagradas, habitualizadas) por el uso lingüístico social; su estructura interna sintáctico-semántica y su combinabilidad como unidades compuestas siguen el sistema gramatical de la lengua» (Zuluaga, 2002b, p. 69). 
recoge distintos capítulos sobre las colocaciones (Veiga, González Pereira y Souto Gómez, 2002), concretamente en el de Ferrando Aramo (2002, p. 100), se presenta como uno de los rasgos de las colocaciones el ser una agrupación arbitraria y no previsible de palabras, en el sentido de que los elementos integrantes de la colocación han sido reunidos por los azares del uso: odio mortal.

Otra muestra, ya posterior, corresponde a la aportación de Vidal y Cabré (2005, p. 898), para quienes uno de los requisitos que cumple una colocación es que la selección o asociación que se produce entre sus constituyentes no es predecible, es totalmente arbitraria: desencadenarse [una] infección. Con total claridad se expresa Travalia (2006, p. 319) al afirmar que las colocaciones son combinaciones lingüísticas arbitrarias resultado del uso por el que optan los hablantes: acaparar la atención. También Muñoz Núñez (2007, p. 192-193) señala como una de las características determinantes en las colocaciones la arbitrariedad a partir de ejemplos (miedo cerval / *pánico cerval) tomados de Alonso Ramos (2002a). Los casos de autores que mantienen el carácter arbitrario de las colocaciones llegan hasta la actualidad, ocurre así con Martos García (2016), quien, para el análisis diacrónico de un conjunto de colocaciones del tipo verbo + sustantivo en función de objeto directo, asume que el colocativo es seleccionado de modo arbitrario por la base9. Asimismo, Corpas Pastor (2017), desde una perspectiva contrastiva, sostiene que: "Collocations are arbitrary, domain-dependent and cohesive lexical patterns which vary from one language to another»: to pay a visit, a compliment, attention, homage en inglés / hacer (una visita), decir (un cumplido) o echar (un piropo), prestar o poner (atención), rendir (homenaje) en español.

Concebir las colocaciones como arbitrarias obliga a deducir que son no motivadas. Sin embargo, no se encuentran afirmaciones explícitas en este sentido por parte de los autores que sostienen su arbitrariedad. En cierto modo, no es el caso de Zuluaga (2002a, p. 105), para quien la fijación arbitraria de los elementos constituyentes de las colocaciones corre pareja con la exclusión semánticamente inmotivada de otros. Por su parte, Blasco Mateo (2002, p. 51) indica de manera expresa que las colocaciones poseen una naturaleza semántica especial: la tendencia a convertirse en signo no motivado por el proceso de lexicalización que las explica, una de cuyas consecuencias es, justamente, la pérdida de su motivación. Asimismo, Martos García (2016, p. 229) se refiere explícitamente al rasgo carencia de motivación lingüística en la preferencia de la base por el colocativo, donde no se da una imposición sintáctica ni semántica, sino una elección dependiente de criterios extralingüísticos entre los que sobresale la consolidación que determina el

\footnotetext{
${ }^{9}$ Con todo, a continuación, esta autora (Martos García, 2016, p. 214) puntualiza que la selección arbitraria del colocativo por la base está hecha en función de vínculos lingüísticos y atendiendo, específicamente, a factores de tipo histórico, cultural o social, pero si existen vínculos lingüísticos y factores históricos, culturales o sociales en la selección del colocativo, resulta dudoso que la arbitrariedad sea un rasgo de las colocaciones.
} 
uso de la comunidad hablante, aceptando, de este modo, la línea teórica inicial de la arbitrariedad en el análisis de las colocaciones ${ }^{10}$.

Con el tiempo, la arbitrariedad de las colocaciones ha sido cuestionada. Fue Bosque quien, de manera expresa, replanteó en un trabajo de 2011 el rasgo arbitrario, interpretado por este autor en el sentido de 'no predecible', rasgo que no es aplicable a la mayoría de las colocaciones desde su perspectiva, pues los colocativos no son elecciones individuales de las bases; al contrario, las bases forman clases semánticas, paradigmas definidos semánticamente, a los que no son ajenos los colocativos, que comparten rasgos semánticos con las bases de las que se predican, tal como ampliamente muestra el diccionario REDES (Bosque, 2004a) ${ }^{11}$, fruto de la concepción teórica sustentada por Bosque al menos desde 2004 (Bosque, 2004b; Bosque, 2004c ${ }^{12}$. La existencia de la propia clase semántica no es, pues, un hecho arbitrario, sino consecuencia del significado de las unidades que la forman: afectar, condicionar, gravitar, incidir, influenciar, influir, marcar, pesar, repercutir son verbos de influencia apropiados para las bases considerablemente, decisivamente, favorablemente, inevitablemente, irremediablemente, etc. (Bosque, 2011, p. XV). Todo lo cual ha dado pie a considerar o bien que las colocaciones son motivadas, o bien que presentan grados de motivación, aunque no siempre se ha explicado qué se entiende por motivación ni en qué consisten o cómo se manifiestan tales grados, tal como podrá comprobarse en el siguiente apartado.

Con alguna excepción (Penadés Martínez, 2015; Penadés Martínez, 2017a), no se han llevado a cabo análisis que especifiquen los rasgos semánticos, compartidos por bases y colocativos, que permiten rechazar la propiedad de la arbitrariedad en las colocaciones. Piénsese, por ejemplo, en el adjetivo clamoroso, que se combina con la clase léxica formada por sustantivos como aplauso, éxito, ovación, triunfo -los cuales designan victoria- porque el rasgo semántico [+ acompañado de gritos] de clamoroso no es incompatible con los significados de los sustantivos ${ }^{13}$, puesto que aplauso se refiere a una aprobación que puede manifestarse con gritos; éxito,

${ }^{10}$ Las perspectivas interlingüística y variacionista, que ya subrayaran Koike (2001) y Castillo (2001), son las pruebas aportadas por Martos García (2016) para justificar la falta de motivación lingüística entre la base y el colocativo.

${ }^{11}$ Aunque sin detallar de manera concreta los rasgos en cuestión.

${ }^{12}$ La postura teórica de Bosque es mantenida, asimismo, por Osorio Olave y Serra Sepúlveda (2012), y por Serra Sepúlveda (2012). Por otra parte, De Miguel (2004) indica que a la propuesta lexicográfica de Bosque (2004a) subyace en parte el modelo teórico del lexicón generativo de Pustejovsky. En este sentido, el análisis en rasgos semánticos del colocativo y la base, y la definición de las colocaciones como estructuras sintagmáticas radiales -análisis y definición bases de este trabajo entroncadas en el estructuralismo europeo- se pueden relacionar con la teoría del lexicón generativo, salvando las diferencias entre la metodología funcional y la generativa.

${ }^{13}$ Así se desprende de las definiciones de estas unidades, y las que se citan en este mismo párrafo, en el diccionario. Esta manera de proceder, tomar la definición lexicográfica de una unidad como correspondiente a su significado, no implica que aquí se identifique definición lexicográfica con significado. 
a una buena aceptación expresada, asimismo, con gritos; ovación, a un aplauso ruidoso, producido también con gritos; y triunfo, a un éxito que se puede mostrar también gritando ${ }^{14}$. Por otra parte, en el tipo de colocación verbo + sustantivo objeto se da este mismo hecho; el colocativo perdonar cuenta entre sus rasgos semánticos con el de [+ hecho reprobable], referido al objeto de perdón, y este mismo rasgo aparece en los sustantivos que, como bases, forman colocaciones con el verbo: delito, desatino, desliz, equivocación, error, pecado. Y un último ejemplo, el adverbio impunemente, colocativo de colocaciones que tienen como base un verbo, presenta, entre otros ${ }^{15}$, el rasgo semántico [+ sin castigo], el cual concuerda con los significados de verbos como asesinar, atacar, dañar, delinquir, golpear, matar, robar, torturar, traficar, violar, dado que todos ellos se refieren a acciones que deben ser objeto de sanción o castigo. Todos estos ejemplos prueban que la relación entre el colocativo y la base no es tan arbitraria como habitualmente se ha venido diciendo, pues existen razones lingüísticas que la explican, el rasgo semántico compartido por los dos elementos de la colocación, el cual determina su coocurrencia en las unidades de la clase de las colocaciones. Además, a partir de aquí es posible definir las colocaciones de dos unidades léxicas como estructuras sintagmáticas radiales en las que un rasgo semántico del colocativo determina su combinación con una clase léxica de unidades, las bases, que comparten ese mismo rasgo (Penadés Martínez, 2015 y 2017a) ${ }^{16}$. El hecho de que una colocación sea una estructura sintagmática implica, además, que entre sus elementos, ya sean todos unidades léxicas ya sean unidades léxicas y fraseológicas, se establecen relaciones sintácticas propias de un grupo nominal, de un grupo adjetival o de un grupo verbal. Y esta particularidad

${ }^{14}$ Por el contrario, si se considera la acepción 3 de clamor en el diccionario (teniendo en cuenta la relación entre clamoroso y clamor): 'Voz lastimosa que indica aflicción o pasión de ánimo' (Real Academia Española, 2014: 547), se puede explicar la motivación de las colocaciones derrota clamorosa o fracaso clamoroso.

${ }^{15}$ Por ejemplo el sema genérico o clasema [+ manera] (Penadés Martínez, 2017a).

${ }^{16}$ Claro está que la falta de arbitrariedad -o de manera contraria, la existencia de motivaciónque se está examinando atañe al significado de las colocaciones. No obstante, tiene incidencia en la combinación de los elementos de la colocación, pues no es posible tratar el significado de una unidad sin referirse a su forma, en este caso a la combinatoria del colocativo y la base, que también es motivada, es decir, no arbitraria. Casos como * pavor cerval frente a miedo cerval y * negar frontalmente frente a oponerse frontalmente no pueden considerarse ejemplos que contradigan la postura que aquí se adopta. Dicho de otro modo, la arbitrariedad de * pánico cerval frente a miedo cerval es aparente, porque la definición de pánico en el diccionario: 'Dicho del miedo o del terror: Extremado o muy intenso [...]' (Real Academia Española, 2014: 1620), acarrea la imposibilidad de que se combine con cerval ('Dicho del miedo: Muy grande o excesivo' (Real Academia Española, 2014: 492), por la tautología a que se daría lugar: el significado del adjetivo cerval ya está incluido en el del sustantivo pánico, hipónimo de miedo definido, consecuentemente, mediante el género 'miedo' más la diferencia específica 'extremado'. En el caso de *negar frontalmente, ni siquiera cabría considerar que es una colocación arbitraria que excluye la combinación que admite un elemento posible del mismo paradigma (oponerse frontalmente), puesto que negar es un verbo de lengua y oponerse, de resistencia u oposición. Cuestión distinta sería si se consideraran negarse y oponerse, ambos verbos de resistencia u oposición que admiten el colocativo frontalmente. 
permite diferenciar una colocación de otras unidades lingüísticas con las que suele relacionarse: las locuciones y los compuestos sintagmáticos ${ }^{17}$.

Posiblemente, cuando se ha hecho referencia a la arbitrariedad impuesta por el uso, se ha adoptado la perspectiva del hablante, que, a la hora de producir, es decir, usar, colocaciones, no conoce la razón o el motivo que conduce a combinar lector empedernido, por ejemplo. Quien ha adoptado más claramente este punto de vista es Alonso Ramos (2017), pues considera que, en el análisis específico de las colocaciones, las bases, efectivamente, se pueden organizar en grupos; sin embargo, desde la perspectiva del hablante que produce una colocación, estas unidades son combinaciones binarias, pues el emisor parte de una unidad léxica única y no de un grupo de unidades léxicas, de manera que no es posible distinguir entre colocativos combinados con un grupo de bases y colocativos combinados con una sola base. Ahora bien, la objeción de Ramos Alonso surge de una perspectiva epistemológica no del todo legítima, pues, si bien es verdad que el investigador, el lingüista, debe explicar la producción lingüística del hablante, la explicación científica no puede hacerse corresponder con la actuación del hablante, sino con el comportamiento de las propias unidades lingüísticas objeto de análisis, el cual demuestra que las colocaciones son deducibles justamente por los rasgos semánticos en los que se corresponden colocativos y bases. Son planos distintos los que atañen al estudioso de la lengua y al hablante. Otra cosa es, claro está, que se quiera investigar los errores en el uso de las colocaciones, por parte de los hablantes no nativos, y la pobreza léxica, por parte de los nativos, $y$, por supuesto, que haya que satisfacer las necesidades de unos y otros, para la producción de colocaciones, con la confección de diccionarios de esta clase de unidades lingüísticas o con la programación de actividades didácticas para su aprendizaje. De ahí el interés en elaborar diccionarios de colocaciones y en reflexionar sobre su enseñanza ${ }^{18}$.

Independientemente de este carácter deducible, no arbitrario, de las colocaciones, para Bosque (2011, p. viii) las colocaciones son arbitrarias en el sentido que Saussure utiliza el término arbitrariedad. Esta afirmación conlleva considerar que el significado de una colocación no está ligado a su significante. Ahora bien, una colocación, frente a una sola unidad léxica, está formada, al menos, por dos unidades lingüísticas, una de las cuales, la base, mantiene el significado y el significante que le corresponden cuando se utiliza como unidad léxica fuera de la propia colocación. En consecuencia, la base, al remitir a un signo lingüístico de la lengua, determina la existencia de una arbitrariedad relativa, o dicho de otro modo, de un grado de motivación. Por lo tanto, las colocaciones son arbitrarias en el sentido de

\footnotetext{
${ }^{17}$ La idea de que las colocaciones no son unidades fraseológicas se va afianzando paulatinamente; véase en este sentido Osorio Olave y Serra Sepúlveda (2012), y Serra Sepúlveda (2012).

${ }^{18}$ Véanse, en este sentido, los capítulos incluidos en las secciones 2. («Lexicographical insights») y 3. («Pedagogical perspectives») de la obra más reciente sobre colocaciones, editada por Torner y Bernal (2017a).
} 
Saussure solo relativamente ${ }^{19}$, lo que implica tener que afirmar que son motivadas en algún grado. Si en leer ávidamente, el verbo leer, base de la colocación, significa lo mismo que cuando no se utiliza en ella, la colocación es motivada por su vinculación, tanto en su significante como en su significado, con la unidad léxica leer. Obsérvese, además, cómo se da una relación sintagmática en la colocación: leer y ávidamente, y una relación asociativa entre la propia colocación, en tanto que signo lingüístico, y el signo leer. Ambas relaciones derivan del carácter relativamente motivado de la colocación.

Así pues, frente a lo que ocurre en el conjunto de los signos lingüísticos, es decir, en las unidades léxicas simples, conjunto en el que pueden registrarse signos absolutamente arbitrarios (haya, pastor, ejemplos de Saussure (1916 [1945, p. 219])), todas las colocaciones son relativamente arbitrarias, o sea, son relativamente motivadas, tomando como referencia la concepción de la arbitrariedad de Saussure, no la visión que deriva esta propiedad del uso lingüístico -punto de vista habitual en el análisis de las colocaciones-, además de ser motivadas en el sentido en que explica Bosque en sus trabajos. Sin embargo, del mismo modo que los autores que se han ocupado del tema de la motivación en Lingüística no se han preocupado por ofrecer explícitamente una definición de motivación que abarque todas las unidades lingüísticas motivadas (Penadés Martínez y Díaz Hormigo, 2008), tampoco en el ámbito más restringido del estudio de las colocaciones se ha precisado con claridad la concepción del término motivación, tal como se observará a continuación en el apartado 3., de ahí la necesidad de ofrecer una definición de este fenómeno y una demostración ulterior de que las colocaciones son motivadas, cuestión que se aborda en 3.1.

\subsection{La motivación en las colocaciones}

Existen trabajos coetáneos de los que caracterizan las colocaciones como arbitrarias que ya se refieren a la motivación de estas unidades lingüísticas. En efecto, Koike (2001, p. 176) habla de la motivación semántica entre los elementos constituyentes de una colocación y, a partir de ella, diferencia entre colocaciones motivadas (barajar las cartas) y colocaciones no motivadas semánticamente, si bien no existe en esta monografía una explicación que aclare qué es motivación y cómo hay que entender la motivación semántica, tampoco se encuentra ejemplos de los que claramente se diga que son colocaciones no motivadas. Por su parte, Ruiz Gurillo

${ }^{19}$ El maestro ginebrino (Saussure, 1916 [1945: 219-222]) diferencia lo que es radicalmente arbitrario, es decir, inmotivado, de lo que es arbitrario relativamente, por tanto relativamente motivado, noción que para Saussure implica el análisis de un signo dado, por consiguiente una relación sintagmática en él, y la evocación de uno o varios términos, o sea, una relación asociativa del signo con otros. 
(2002) concluye que las colocaciones pueden delimitarse, además de por otros rasgos, por el de la motivación, entendida como trasparencia paradigmática de la unidad cuyo significado puede ser interpretado ${ }^{20}$. Más recientemente, Sánchez Rufat (2010) también se refiere a la motivación al clasificar las combinaciones léxicas basándose, precisamente, en la motivación de su restricción. En el trabajo citado motivación se equipara a explicación en el sentido de que, en ciertas combinaciones léxicas, existen restricciones de las que se desconoce de qué son producto, cuál es la motivación o explicación semántica que justifica la diferente preferencia léxica, como ocurre con ecuestre y equino, pues el primer adjetivo selecciona a escultura, retrato, monumento, arte o figura, mientras que el segundo se combina con peste, gripe, ganado, animal o reproducción, pese a su aparente identidad de significado (Sánchez Rufat, 2010, p. 298) ${ }^{21}$. Asimismo, Barrios Rodríguez (2016), aunque sin explicar el concepto, alude a la motivación, concretamente a la motivación semántica que subyace a los verbos soporte, en la cual se apoya para clasificarlos con vistas a la enseñanza de un conjunto de colocaciones que los contienen a estudiantes de español como lengua extranjera. Así, según esta autora (Barrios Rodríguez, 2016, p. 6), en las colocaciones que expresan emoción es frecuente recurrir al verbo sentir, como en sentir alegría / tristeza / emoción; en las que expresan movimiento es frecuente recurrir al verbo dar, como en dar un brinco / bote / salto ${ }^{22}$.

Por otra parte, investigadores que adoptan una perspectiva cognitiva, junto con cuyos intereses están centrados en la enseñanza de la L2, especialmente del inglés, han subrayado el carácter motivado de las colocaciones con más énfasis que en los autores que se ocupan del español, aunque sin explicar cómo conciben la motivación. En efecto, Radden y Panther, editores de una obra de orientación cognitiva

${ }^{20}$ No acaba de quedar claro, sin embargo, qué se entiende por trasparencia paradigmática, pues los ejemplos analizados como colocaciones en el estudio: lógica aplastante, afirmación gratuita, rabiosa actualidad e inflación galopante, no se ponen en relación paradigmática con ningún otro elemento ni, por supuesto, puede considerarse que se relacionan entre sí de ese modo.

${ }^{21}$ Con todo, el objetivo de esta autora, proponer un modelo de clasificación de las combinaciones léxicas basado en la motivación de su restricción, no acaba de alcanzarse porque no queda claro cómo el concepto de motivación utilizado permite diferenciar entre combinaciones léxicas que constituyen restricciones no sistemáticas frente a las que son restricciones sistemáticas, y dentro de las primeras entre combinaciones léxicas que son unidades fraseológicas (paremias, fórmulas rutinarias y locuciones) frente a las que son preferencias léxicas (cámara mortuoria o coche fúnebre); por su parte, en las restricciones sistemáticas, no se sabe cómo la motivación de su restricción permite deslindar las restricciones sistemáticas intralingüísticas (colocaciones en sentido restringido, como lavar la imagen o lavar la reputación) de las extralingüísticas (lavar los platos o lavar el coche). Dicho de otro modo, el análisis de Sánchez Rufat (2010) suscita interrogantes como ¿existe alguna motivación para producir lavar la imagen? y, si la hay, ¿̇cuál es?, ¿en qué consiste?

${ }^{22}$ No obstante, el propio trabajo de Barrios Rodríguez (2016: 6-7) muestra que dar se combina con sustantivos de comunicación (dar una clase), de sentimientos (dar alegría) y de percepción (dar la sensación), asistencia (dar apoyo), estímulo (dar ánimo) y autorización (dar un permiso). Además, los sustantivos de sentimiento también se combinan con tener (tener alegría), de modo que no se acaba de comprender el sentido en que se toma motivación semántica ni su incidencia en la clasificación de las colocaciones con verbo soporte. 
sobre la motivación en lingüística (Radden y Panther, 2004a), tema al que aportan también sus reflexiones, reconocen que en la lingüística actual el término motivación suele ser autosuficiente y que falta una teoría unificada de la motivación (Radden y Panther, 2004b, p. 2). A pesar de ello, cuando proporcionan su definición: "A linguistic unit (target) is motivated if some of its properties are shaped by a linguistic source (form and/or content) and language-independent factors" (Radden y Panther, 2004b, p. 4), lo que ofrecen, realmente, no es una explicación de este fenómeno lingüístico, sino de su resultado, es decir, no explican qué es la motivación, sino cuál es la condición para que exista una unidad lingüística motivada. Incomprensiblemente, proceden así, aunque, de manera previa, han analizado la definición de motivación en psicología y han establecido una serie de criterios que, según ellos, deben servir de base para su definición en lingüística: 1) la forma y/o el contenido de una unidad lingüística deben ser los desencadenantes de un proceso de motivación; 2) la acción recíproca de una fuente lingüística y de factores independientes del lenguaje determinan un proceso de motivación; 3) todo proceso de motivación en el lenguaje afecta a la conducta lingüística del hablante; 4) la conducta lingüística puede fijarse en estructuras lingüísticas recurrentes y estables cuyo resultado final sea una unidad lingüística motivada. En esta misma línea de la lingüística cognitiva, pero ahora aplicada a la enseñanza del vocabulario y la fraseología del inglés como lengua extranjera, Walker (2008) rechaza la arbitrariedad de las colocaciones y prueba su motivación, aunque sin definir tampoco qué entiende por tal.

En el trabajo de Bosque (2011) al que antes se ha hecho referencia, este lingüista no relaciona expresamente la falta de arbitrariedad de las colocaciones con la motivación, sino con la particularidad de ser deducibles; sin embargo, en la actualidad, concibe las colocaciones como unidades motivadas, deducibles o composicionales (cerrar, sellar herméticamente), independientemente de la necesidad de establecer el grado en que lo son (Bosque, 2017). La influencia de sus trabajos previos se manifiesta en otros capítulos de la más reciente monografía sobre colocaciones del español (Torner y Bernal, 2017a), en la que de manera reiterada se encuentran referencias al carácter semánticamente motivado de las colocaciones (Alonso Ramos, 2017; Torner y Bernal, 2007b; Higueras, 2017).

Puede concluirse, pues, de este apartado que en el análisis de las colocaciones se ha producido un vuelco que ha conducido desde su consideración como arbitrarias a entenderlas a partir de la propiedad contraria: la motivación. Sin embargo, es necesaria una concepción explícita de este rasgo y una demostración desde ella de que existen colocaciones motivadas, independientemente de que, por el momento, no tengamos analizadas todas las unidades del español pertenecientes a esta clase. 


\subsection{Colocaciones motivadas}

En Penadés Martínez y Díaz Hormigo (2008) se ha definido motivación como la relación causal entre el significante y el significado de una unidad linguística, entendiendo causal en el sentido de que hay una razón o un motivo para asociar el significado de un signo a un determinado significante y no a otro, razón o motivo que surge de la vinculación de la unidad lingüística que resulta motivada ya sea con otras unidades de la lengua (motivación morfológica), ya sea con la realidad extralingüística (motivación fonética) o ya sea con la realidad extralingüística y con otras unidades de la lengua simultáneamente (motivación semántica). Así, en combinaciones de unidades que corresponden a la clase de las locuciones, $a$ borbotones sería un claro ejemplo de locución con motivación fonética, pues la relación entre el significante (está constituida por la palabra borbotón como elemento principal) y el significado ('Referido a un líquido: Agitándose al hervir, al brotar o al caer en chorro' (Seco, Andrés y Ramos, 2004, p. 199)) se origina en un fenómeno de la realidad: el ruido producido por el agua al nacer o al hervir. Por otra parte, existen numerosas locuciones adverbiales, del tipo en concreto y en exclusiva, y adjetivas, como de costumbre y de impresión, cuya motivación es morfológica, producida por su relación con los signos lingüísticos concreto, exclusivo, costumbre e impresión; son tanto el significante como el significado de estos signos los que desencadenan la motivación de las respectivas unidades fraseológicas, vinculadas a ellos desde el punto de vista de su combinatoria formal y por su significado no idiomático. Por último, la relación causal entre el significante y el significado de una unidad fraseológica puede surgir, asimismo, de su vinculación con la realidad extralingüística y, a la vez, con otras unidades de la lengua; se explica así la existencia de fraseologismos que responden a la motivación semántica, pues en ellos se manifiesta la vinculación de un signo con otro signo con el que coincide desde el punto de vista del significante y que designa en la realidad un objeto o concepto con el que lo designado por el primero presenta o una relación de analogía o semejanza, o una relación de contigüidad espacial o temporal, o alguna otra relación de carácter extralingüístico; de este modo, locuciones como echar raices, 'establecerse en un lugar' (Penadés Martínez, en preparación), o dar en el clavo, 'acertar plenamente en una cuestión' (Penadés Martínez, en preparación), pueden explicarse por la asociación entre las respectivas combinaciones literales de palabras y las combinaciones fraseológicas, además del apoyo que la realidad presta por la semejanza entre la planta que echa raíces y la persona que se establece en un lugar o el parecido entre la acción de dar en un clavo con un martillo y el proceso mental de acertar, adivinar o descubrir.

Si pasamos al plano de las colocaciones ${ }^{23}$, no parece que la motivación foné-

${ }^{23}$ Lo que no debe interpretarse como que las colocaciones se consideran aquí unidades fraseo- 
tica se dé en unidades pertenecientes a esta clase, a menos que se documenten ejemplos que la prueben ${ }^{24}$. Por el contrario, sí puede considerarse que existe motivación morfológica en todas las colocaciones, dado que, de manera hasta cierto punto análoga a derivados y compuestos ${ }^{25}$, su estructura es transparente y su significado se deduce, al menos en parte, del elemento que constituye la base de la colocación. La vinculación de las colocaciones con otras unidades de la lengua, a partir, pues, del elemento base, determina su motivación morfológica. Y ello ocurre tanto en las colocaciones formadas con un verbo soporte y un sustantivo (dar un paseo se vincula con paseo, hacer una pregunta, con pregunta ${ }^{26}$, como en las colocaciones de los distintos tipos establecidos: error garrafal lo hace con error; conciliar el sueño, con sueño; afirmar rotundamente, con afirmar; perdidamente enamorado, con enamorado.

También es posible afirmar que, además de la motivación morfológica, en cuanto colocaciones que son, hay motivación semántica en las examinadas ya en Penadés Martínez (2015) y Penadés Martínez (2017a), concretamente en las colocaciones complejas ${ }^{27}$ formadas con la locución sobre ruedas en combinación con los verbos ir, marchar, discurrir, deslizarse y seguir, todos ellos verbos, en principio, de movimiento, pero que cuentan también con una acepción relativa a denotar que algo funciona o se desarrolla. La formación de estas colocaciones complejas tiene que ver con el proceso metafórico que permite proyectar el dominio concreto del movimiento real sobre el dominio abstracto del desarrollo de algo, con lo que se crea la metáfora LA MARCHA DE UN ASUNTO ES LA MARCHA DE UN AUTOMÓVIL ${ }^{28}$. De este modo, en una colocación como ir sobre ruedas existe motivación semántica porque está vinculada con otras unidades de la lengua (las propias unidades léxi-

lógicas, la relación entre las locuciones y las colocaciones, establecida en este trabajo para tratar la motivación de estas dos clases, viene dada por el simple hecho de constituir ambas combinaciones de palabras. Las colocaciones no son unidades fraseológicas, básicamente, porque entre sus elementos constituyentes se establece una relación sintáctica, lo que no se da en las locuciones, y porque el elemento base de la colocación tiene un significado autónomo, el mismo que tiene cuando no es usado en ella, a diferencia de las locuciones. Las colocaciones, más bien, son unidades de la interfaz léxico-sintaxis (Penadés Martínez, 2017a).

${ }^{24}$ La motivación fonética, que corresponde a las onomatopeyas, es uno de los tipos establecidos por Ullmann ([1962] 1976: 93-106) y por Guiraud ([1955] 1969: 23-29).

${ }^{25}$ Justamente, la motivación morfológica es el tipo que corresponde a estas dos clases de unidades, derivados y compuestos, según los autores citados en la nota anterior.

${ }^{26}$ No en vano las colocaciones con verbo soporte han recibido, entre otras denominaciones, las de lexemas verbales compuestos y verbos compuestos.

${ }^{27}$ El término colocación compleja es utilizado por Koike (2001: 55-60) para referirse a la coocurrencia léxica existente entre una locución y una unidad léxica simple cuando entre ambos constituyentes se establece una restricción combinatoria. Por otra parte, en Koike (2005) se amplía el alcance del término al considerar que una unidad léxica simple se combina con una unidad fraseológica y, asimismo, con otra unidad léxica compleja (dividir, repartir o distribuir en partes iguales).

${ }^{28}$ Para explicar la formación de sobre ruedas es necesario acudir también al proceso metonímico de PARTE POR TODO. 
cas que la constituyen) y, además, con la realidad extralingüística por la relación conceptual que la metáfora establece entre la marcha de un automóvil (dominio fuente) y la marcha de un asunto (dominio meta).

De manera inversa, en el caso de las también colocaciones complejas formadas con saber, sonar, oler o retumbar, paradigma de los verbos de percepción sensitiva, y la locución adverbial a gloria, la situación abstracta de bienaventuranza, incluso de felicidad, que se obtendría estando en el cielo, en el Paraíso, en la gloria, contemplando a Dios, se proyecta, en tanto que dominio meta, sobre el fenómeno sensorial, concreto por tanto, de degustar u oler un alimento muy sabroso: saber u oler a gloria, o de percibir un sonido muy agradable: sonar o retumbar a gloria (fenómenos sensoriales que constituyen el dominio meta), así que ahora se está ante la metáfora UNA PERCEPCIÓN SENSORIAL AGRADABLE ES LA GLORIA CELESTIAL, con lo que, de nuevo, existe en estas colocaciones motivación semántica, es decir, una relación causal entre su significante y su significado surgida de la vinculación de la colocación (saber a gloria) con otras unidades de la lengua (saber y gloria) y con la realidad extralingüística a partir de la semejanza, analogía o identidad que conceptualmente se establece entre los dominios fuente y meta.

Por otra parte, la relación entre significante y significado de una unidad lingüística motivada puede ser percibida como causal, es decir, como fruto de la existencia de una razón o un motivo que lleva a asociar el significado del signo a un determinado significante y no a otro, solo desde el plano del investigador, del lingüista, con exclusión del hablante. Aplicando esta perspectiva a las colocaciones, estaríamos ante unidades de esta clase diacrónicamente motivadas, aunque en la actualidad no se perciba la motivación de los dos elementos constituyentes de la colocación, no solo la motivación de la base, sin el correspondiente estudio. Serían, pues, datos de naturaleza diacrónica, resultado del análisis de los estudiosos, los que darían cuenta de que la base y el colocativo de una colocación, en tanto que unidades que la forman y constituyen su significante, no son arbitrarias en relación con el significado de la propia colocación, sino motivadas, aunque la motivación de ambos elementos no sea percibida ni comprendida por el hablante en una primera aproximación, es decir, sin un conocimiento de los datos diacrónicos que la justifican ${ }^{29}$.

Para el análisis de la motivación en el ámbito de la Lingüística también puede tomarse como referencia al hablante. Esta perspectiva conduce: 1) a explicar los signos lingüísticos motivados por la acción de la etimología popular ${ }^{30} ; 2$ ) a analizar la modificación creativa de las locuciones, que puede originar, por la acción individual de un hablante, su interpretación literal, lo cual se produce al asociar

${ }^{29}$ En Penadés Martínez (2015) y Penadés Martínez (2017a) está explicada la motivación diacrónica de las colocaciones perdidamente enamorado y comer opiparamente / comida opipara.

${ }^{30}$ Véase, por ejemplo, el interesante estudio de García Manga (2011). 
una locución -es decir, unos significantes con un significado, en principio, idiomático- a un significado literal, motivado por el significado de los elementos que componen la locución en tanto que combinación de palabras ${ }^{31}$; y 3) a considerar que la interpretación o comprensión de las colocaciones sea más factible que su producción por parte del hablante, con los consecuentes errores en el uso de las colocaciones $^{32}$, en el proceso de codificación, por la imposibilidad de producirlas en ocasiones, de ahí el sentido de la afirmación de Corpas Pastor (2017) acerca de que son arbitrarias desde un punto de vista contrastivo o interlingüístico, si bien, como se ha mostrado en este trabajo, son relativamente arbitrarias, es decir, motivadas, desde el punto de vista intralingüístico.

\section{CONCLUSIONES}

Desde una perspectiva teórica, y realizando un recorrido hasta cierto punto historiográfico, en este trabajo 1) se ha mostrado que, en general, existe una falta de precisión terminológica por parte de los autores que se refieren a la arbitrariedad y la motivación de las colocaciones, dado que no parten de una definición concreta de los términos arbitrariedad y motivación.

2) Se ha comprobado cómo, en un primer momento, la arbitrariedad en las colocaciones se ha entendido como la falta de explicación en la aparición conjunta de las dos unidades, base y colocativo, que constituyen una colocación. Solo el uso lingüístico se toma como justificación de las restricciones que la base impone al colocativo. Esta visión teórica de las colocaciones no puede sustentarse en la actualidad principalmente como consecuencia de los estudios de Bosque, quien ha demostrado que un colocativo no es una elección individual de una base. En este sentido, este artículo ofrece distintos ejemplos de cómo un rasgo semántico de un colocativo es compartido por una clase léxica de bases que se combinan con él.

3) Asimismo, en el artículo se sostiene que todas las unidades de la clase de las colocaciones son relativamente arbitrarias, es decir, son motivadas tomando una colocación en conjunto, como un signo lingüístico, y aplicando para su examen la concepción saussureana de lo arbitrario relativo, pues las colocaciones admiten un análisis sintagmático (base + colocativo), presentan sin lugar a dudas elementos formativos (de nuevo, base + colocativo) y un significado autónomo en, al menos, uno de ellos (la base). Si todas las colocaciones son relativamente arbitrarias, por tanto motivadas, frente a las unidades léxicas, donde existen signos lingüísticos arbitrarios y motivados, la propiedad de la motivación sirve para caracterizarlas en (2014)

${ }^{31}$ Pueden encontrarse ejemplos de modificación creativa de las locuciones en Penadés Martínez

${ }^{32}$ Hay ejemplos de errores en el uso de las colocaciones en Vincze (2015). 
relación con las unidades léxicas, que pueden ser, como se ha indicado, tanto no motivadas (arbitrarias) como motivadas

4) Desde la definición de motivación como relación causal entre el significante y el significado de una unidad lingüística, entendiendo causal en el sentido de que hay una razón o un motivo para asociar el significado de un signo a un determinado significante y no a otro, se ha comprobado que es posible establecer distinciones en las colocaciones en función de la clase de motivación que presentan (solo morfológica o morfológica y semántica simultáneamente).

5) En el análisis de la motivación de las colocaciones, es posible diferenciar entre colocaciones diacrónica y sincrónicamente motivadas, frente a las motivadas solo sincrónicamente; las primeras lo son desde la perspectiva del investigador, las segundas desde el punto de vista del hablante, que solo puede establecer la vinculación de un elemento de la colocación, la base, con otra unidad de la lengua, no ofrecer una explicación lingüística de la coocurrencia de la base y el colocativo.

6) De las cuestiones abordadas en este artículo también se desprenden conclusiones de naturaleza aplicada en relación con la práctica lexicográfica y la didáctica de la lengua. En efecto, si los significados de la base y el colocativo de una colocación comparten rasgos semánticos, la definición del significado de uno y otro elemento en un diccionario no podrá ignorar esta circunstancia, de modo que la labor del diccionarista, en el tratamiento lexicográfico del significado de las colocaciones y de sus elementos constituyentes, estará condicionada por esta perspectiva teórica, ya sea en un diccionario general de lengua, en un diccionario específico de combinatoria léxica, en un diccionario de orientación didáctica o en un diccionario bilingüe. Y ello independientemente de todos los aspectos particulares que estos tipos de diccionarios deben abordar en relación con las colocaciones ${ }^{33}$.

7) Por último, la enseñanza-aprendizaje de las colocaciones en los ámbitos de la L1 y la L2 también se ve condicionada por la asunción de los principios teóricos desarrollados en este artículo: la base y el colocativo comparten algún rasgo semántico y las colocaciones son motivadas. Ambos principios constituyen un apoyo mnemotécnico para el aprendiz de la lengua ${ }^{34}$, que no se ve obligado a memorizar pares de unidades lingüísticas que van juntas aparentemente sin ton ni son. Al contrario, es posible hacerle ver la relación semántica entre los elementos de una clase léxica, verbos por ejemplo, y la sintáctico-semántica entre ellos y otra unidad lingüística, un adverbio, por ejemplo, en una colocación en la que los verbos son las bases y el adverbio, el colocativo.

${ }^{33}$ Véanse, en este sentido, los análisis de Maldonado (2017); Torner y Bernal (2017b); Corpas Pastor (2017).

${ }^{34}$ Por supuesto, no son los únicos que rigen la enseñanza-aprendizaje de esta clase de unidades lingüísticas, tal como se desarrolla en Penadés Martínez (2017b). 


\section{REFERENCIAS}

Alonso Ramos, Margarita. 1994-1995. Hacia una definición del concepto de colocación: de J. R. Firth a I. A. Mel'čuk. Revista de Lexicografía, I, 9-28.

Alonso Ramos, Margarita. 2002a. Colocaciones y contorno de la definición lexicográfica. Lingüística Española Actual, XXIV/1, 63-96.

Alonso Ramos, Margarita. 2002b. Presentación del Diccionario de colocaciones y marcadores del español: estructura y objetivos. En María Dolores Muñoz Núñez, Ana Isabel Rodríguez-Piñero Alcalá, Gérard Fernández Smith y Victoria Benítez Soto (eds.) IV Congreso de Lingüistica General, Cádiz, del 3 al 6 de abril de 2000. Volumen II. Comunicaciones. Cádiz, España: Área de Lingüística General de la Universidad de Cádiz / Servicio de Publicaciones de la Universidad de Cádiz / Servicio de Publicaciones de la Universidad de Alcalá, 47-61.

Alonso Ramos, Margarita. 2017. Can collocations be deduced? A lexically-driven analysis from the perspective of language production. En Sergi Torner y Elisenda Bernal (eds.) Collocations and other Lexical Combinations in Spanish. Theoretical, Lexicographical and Applied Perspectives. London, Reino Unido: Routledge, 21-40.

Barrios Rodríguez, María Auxiliadora. 2016. Propuesta metodológica para la enseñanza de las colocaciones de verbo soporte a estudiantes anglófonos: estudio empírico. marcoELE. Revista de Didáctica Español como Lengua Extranjera, 23, 1-13. ISSN 1885-2211. Disponible en http://marcoele.com/colocaciones-verbales/. [Consulta: 28/2/17).

Blasco Mateo, Esther. 2002. La lexicalización y las colocaciones. Lingüística Española Actual, XXIV/1, 35-61.

Bosque, Ignacio. (dir.) 2004a. Las palabras en su contexto. REDES. Diccionario combinatorio del español contemporáneo. Madrid, España: Ediciones SM.

Bosque, Ignacio. 2004b. La dirección en los diccionarios combinatorios y el problema de la selección léxica. En Teresa Cabré (ed.) Lingüística Teòrica: anàlisi i perspectives, I, Catalan Journal of Linguistics Monographies, 13-58. ISBN 84490-2374-2. Disponible en http://filcat.uab.es/clt/publicacions/coleccions/ monografies/pdf/LT-I-Bosque.pdf. [Consulta: 9/2/2017].

Bosque, Ignacio. 2004c. Combinatoria y significación. Algunas reflexiones. En Ignacio Bosque (dir.) Redes. Diccionario combinatorio del español contemporáneo. Madrid, España: SM, LXXVII-CLXXIV.

Bosque, Ignacio. 2011. Deducing collocations. En Igor Boguslavsky y Leo Wanner (eds.), Proceedings of the $5^{\text {th }}$ International Conference on the Meaning-Text Theory, Barcelona, September 8-9, 2011. Barcelona, España, vi-xxiii. ISBN 978-84-615-1. Disponible en http://olst.ling.umontreal.ca/pdf/proceedingsMTT2011.pdf. [Consulta: 19/2/2017].

Bosque, Ignacio. 2017. On the conceptual bases of collocations: restricted adverbs 
and lexical selection. En Sergi Torner y Elisenda Bernal (eds.) Collocations and other Lexical Combinations in Spanish. Theoretical, Lexicographical and Applied Perspectives. London, Reino Unido: Routledge, 9-20.

Castillo, Ma Auxiliadora. 2001. Colocaciones léxicas y variación lingüística: implicaciones didácticas. Lingüistica Española Actual, XXIII/1, 133-143.

Corpas Pastor, Gloria. 1996. Manual de fraseología española. Madrid, España: Gredos.

Corpas Pastor, Gloria. 1998. Expresións fraseolóxicas e colocacións: clasificación. En Xesús Ferro Ruibal (coord.) Actas do I Coloquio Galego de Fraseoloxía. Centro Ramón Piñeiro, Santiago de Compostela, 1997. Vigo, España: Xunta de Galicia, 31-61.

Corpas Pastor, Gloria. 2001. Apuntes para el estudio de la colocación. Lingüistica Española Actual, XXIII/1, 41-56.

Corpas Pastor, Gloria. 2017. Collocations in e-bilingual dictionaries: from underlying theoretical assumptions to practical lexicography and translation issues. En Sergi Torner y Elisenda Bernal (eds.) Collocations and other Lexical Combinations in Spanish. Theoretical, Lexicographical and Applied Perspectives. London, Reino Unido: Routledge, 173-199.

De Miguel, Elena. 2004. La Teoría del Lexicón Generativo. En Elena de Miguel (coord.) Panorama de la lexicología. Barcelona, España: Ariel, 337-370.

Ferrando Aramo, Verónica. 2002. Colocaciones y compuestos sintagmáticos: dos fenómenos léxicos colindantes. En Alexandre Veiga, Miguel González Pereira y Montserrat Souto Gómez (eds.) Léxico y gramática. Lugo, España: Tris Tram, 99-107.

García Manga, Ma del Carmen. 2011. La creación discursiva de etimologismos basada en aspectos socio-culturales. Lingüistica en la Red, IX, 1-23. ISSN 1697-0780. Disponible en http://www.linred.es/articulos.html. [Consulta: 26/1/2017].

García-Page, Mario. 2001. El adverbio colocacional. Lingüistica Española Actual, XXIII/1, 89-105.

Guiraud, Pierre. [1955] 1969. La sémantique (9a edic.). Paris, Francia: Presses Universitaires de France.

Higueras García, Marta. 2006a. Estudio de las colocaciones léxicas y su enseñanza en español como lengua extranjera. Málaga, España: ASELE / Ministerio de Educación y Ciencia, Secretaría General de Educación.

Higueras García, Marta. 2006b. Las colocaciones y su enseñanza en la clase de ELE. Madrid, España: Arco/Libros.

Higueras García, Marta. 2011. Lexical collocations and the learning of Spanish as a foreign language. En José Luis Cifuentes Honrubia y Susana Rodríguez Rosique (eds.) Spanish Word Formation and Lexical Creation. Amsterdam / Philadelphia, Dinamarca / USA: John Benjamins Publishing Company, 439-463. 
Higueras García, Marta. 2017. Pedagogical principles for the teaching of collocations in the foreign language classroom. En Sergi Torner y Elisenda Bernal (eds.) Collocations and other Lexical Combinations in Spanish. Theoretical, Lexicographical and Applied Perspectives. London, Reino Unido: Routledge, 250266.

Koike, Kazumi. 2001. Colocaciones léxicas en el español actual: estudio formal y léxico-semántico. Alcalá de Henares, España: Universidad de Alcalá / Takushoku University.

Koike, Kazumi. 2005. Colocaciones complejas en el español actual. En Ramón Almela, Estanislao Ramón Trives y Gerd Wotjak (eds.) Fraseología contrastiva con ejemplos tomados del alemán, español, francés e italiano. Murcia, España: Servicio de Publicaciones de la Universidad de Murcia, 169-184.

Maldonado, Concepción. 2017. Word combinations in general dictionaries. En Sergi Torner y Elisenda Bernal (eds.) Collocations and other Lexical Combinations in Spanish. Theoretical, Lexicographical and Applied Perspectives. London, Reino Unido: Routledge, 139-156.

Martos García, Paula. 2016. Sobre la aplicación de los criterios de análisis de colocaciones verbo + sustantivo ${ }_{\mathrm{CD}}$ a materiales históricos. Estudios de Lingüistica. Universidad de Alicante, 30, 213-238.

Mounin, George. 1979. Historia de la lingüistica. Desde los origenes al siglo XX (1a edic., 3a reimp.). Madrid, España: Gredos.

Muñoz Núñez, Ma Dolores. 2007. Colocaciones y restricciones léxicas: criterios de diferenciación. En Virginia Bertolotti, Serrana Caviglia, Sylvia Costa, Mariela Grassi, Marisa Malcuori y Ma Dolores Muñoz Núñez Estudios de lingüística hispánica. Cádiz, España: Servicio de Publicaciones de la Universidad de Cádiz, 177-198.

Osorio Olave, Gabriela y Serra Sepúlveda, Susana. 2012. Colocaciones, compuestos sintagmáticos y locuciones nominales: hacia un intento de delimitación conceptual. Lenguas Modernas, 39, 103-116.

Penadés Martínez, Inmaculada. 2014. Fixación estrutural e desautomatización das locucións. Cadernos de Fraseoloxía Galega, 16, 273-301.

Penadés Martínez, Inmaculada. 2015. Las colocaciones del tipo verbo más locución adverbial: implicaciones teóricas y aplicadas. Lingüistica en la Red, XII, 1-13. ISSN 1697-0780. Disponible en http://www.linred.es/articulos_pdf/ LR_articulo_05042015.pdf [Consulta: 24/2/2017].

Penadés Martínez, Inmaculada. 2017a. El concepto de colocación a la luz de las colocaciones del tipo verbo más locución adverbial. En Miguel Casas Gómez y Martin Hummel (eds.) Semántica léxica, volumen monográfico de RILCE. Revista de Filologia Hispánica, 33, 3, 963-991.

Penadés Martínez, Inmaculada. 2017b. La enseñanza de las unidades fraseológicas. En Ana María Cestero Mancera e Inmaculada Penadés Martínez (eds.) 
Manual del profesor de ELE. Alcalá de Henares, España: Servicio de Publicaciones de la Universidad de Alcalá, 311-355.

Penadés Martínez, Inmaculada. En preparación. Diccionario de locuciones idiomáticas del español actual.

Penadés Martínez, Inmaculada y Díaz Hormigo, Ma Tadea. 2008. Hacia la noción lingüística de motivación. En María Álvarez de la Granja (ed.) Lenguaje figurado y motivación. Frankfurt am Main, Alemania: Peter Lang, 51-68.

Perl, Matthias. 1975. Semantische Selektionsbeschränkungen (semantische Valenz) und sprachlich usuell bedingte Restriktionen. Linguistische Arbeitsberichte, 11, 48-51.

Radden, Gunter y Panther, Klaus-Uwe. 2004a. Studies in Linguistic Motivation. Berlin, Alemania: Mouton de Gruyter.

Radden, Gunter y Panther, Klaus-Uwe. 2004b. Introduction: reflections on motivation. En Gunter Radden y Klaus-Uwe Panther (eds.) Studies in Linguistic Motivation. Berlin, Alemania: Mouton de Gruyter, 1-46.

Real Academia Española. 2014. Diccionario de la lengua española (23a edic.). Barcelona, España: Espasa Libros.

Ruiz Gurillo, Leonor. 2002. Compuestos, colocaciones, locuciones: intento de delimitación. En Alexandre Veiga, Miguel González Pereira y Montserrat Souto Gómez (eds.) Léxico y gramática. Lugo, España: Tris Tram, 325-339.

Sánchez Rufat, Anna. 2010. Apuntes sobre las combinaciones léxicas y el concepto de colocación. Anuario de Estudios Filológicos, XXXIII, 291-306.

Saussure, Ferdinand de. [1916] 1945. Curso de lingüistica general. Publicado por Charles Bally y Albert Sechehaye. Con la colaboración de Albert Riedlinger. Traducción, prólogo y notas de Amado Alonso. Buenos Aires, Argentina: Losada.

Seco, Manuel, Andrés, Olimpia y Ramos, Gabino. 2004. Diccionario fraseológico documentado del español actual. Locuciones y modismos españoles. Madrid, España: Aguilar.

Serra Sepúlveda, Susana. 2012. Gramática y diccionario: contornos, solidaridades léxicas y colocaciones en lexicografía española contemporánea. Tesis doctoral, Madrid, España: Universidad Complutense de Madrid.

Torner, Sergi y Bernal, Elisenda (eds.). 2017a. Collocations and other Lexical Combinations in Spanish. Theoretical, Lexicographical and Applied Perspectives. London, Reino Unido: Routledge.

Torner, Sergi y Bernal, Elisenda. 2017b. Collocations in learner's dictionaries. En Sergi Torner y Elisenda Bernal (eds.) Collocations and other Lexical Combinations in Spanish. Theoretical, Lexicographical and Applied Perspectives. London, Reino Unido: Routledge, 157-172.

Travalia, Carolina. 2006. Las colocaciones implícitas. Estudios de Lingüistica. Universidad de Alicante, 20, 317-332. 
Ullmann, Stephen. [1962] 1976. Semántica. Introducción a la ciencia del significado (2da edic.). Madrid, España: Aguilar.

Veiga, Alexandre, González Pereira, Miguel y Souto Gómez, Montserrat (eds.). 2002. Léxico y gramática. Lugo, España: Tris Tram.

Vidal, Vanesa y Cabré, M. Teresa. 2005. La combinatoria léxica en la enseñanza y aprendizaje de lenguas para propósitos específicos. En Ma Auxiliadora Castillo et alii (coords.) Las gramáticas y los diccionarios en la enseñanza del español como segunda lengua: deseo y realidad. Actas del XV Congreso Internacional de ASELE, Sevilla, 22-25 de septiembre de 2004. Sevilla, España: Universidad de Sevilla, Secretariado de Publicaciones, 896-902.

Vincze, Orsolya. 2015. Learning Multiword Expressions from Corpora and Dictionaries. Tesis de doctorado. A Coruña, España: Universidade da Coruña.

Walker, Crayton. 2008. Factors which influence the process of collocation. En Frank Boers y Seth Lindstromberg (eds.) Cognitive Linguistic Approaches to Teaching Vocabulary and Phraseology. Berlin, Alemania: Mouton de Gruyter, 291-308.

Zuluaga, Alberto. 1998. Sobre fraseoloxismos e fenómenos colindantes. En Xesús Ferro Ruibal (coord.) Actas do I Coloquio Galego de Fraseoloxía. Centro Ramón Piñeiro, Santiago de Compostela, 1997. Vigo, España: Xunta de Galicia, 15-30.

Zuluaga, Alberto. 2002a. Los «enlaces frecuentes» de María Moliner. Observaciones sobre las llamadas colocaciones. Lingüistica Española Actual, XXIV/1, 97-114.

Zuluaga, Alberto. 2002b. Los «enlaces frecuentes» de María Moliner. Observaciones sobre las llamadas colocaciones. Philologie im Netz, 22, 56-74. ISSN 1433-7177. Disponible en http://web.fu-berlin.de/phin/phin22/p22t3.htm. [Consulta: 19/2/2017]. 